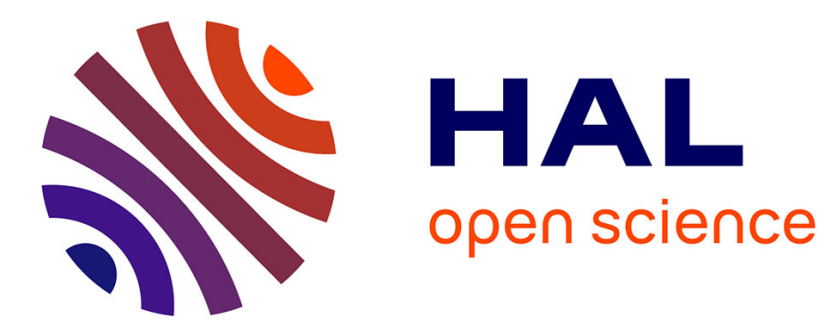

\title{
Quelques effets narratifs de la pseudépigraphie jérémienne
}

\author{
Elena Di Pede
}

\section{To cite this version:}

Elena Di Pede. Quelques effets narratifs de la pseudépigraphie jérémienne. Études Théologiques et Religieuses, 2016, 91 (4), p. 599-605. 10.3917/etr.0914.0599 . hal-02924452

\section{HAL Id: hal-02924452 \\ https://hal.univ-lorraine.fr/hal-02924452}

Submitted on 7 Feb 2022

HAL is a multi-disciplinary open access archive for the deposit and dissemination of scientific research documents, whether they are published or not. The documents may come from teaching and research institutions in France or abroad, or from public or private research centers.
L'archive ouverte pluridisciplinaire HAL, est destinée au dépôt et à la diffusion de documents scientifiques de niveau recherche, publiés ou non, émanant des établissements d'enseignement et de recherche français ou étrangers, des laboratoires publics ou privés. 


\title{
QUELQUES EFFETS NARRATIFS DE LA PSEUDÉPIGRAPHIE JÉRÉMIENNE
}

\author{
Elena Di Pede
}

Institut protestant de théologie | «Études théologiques et religieuses »

2016/4 Tome 91 | pages 599 à 605

ISSN 0014-2239

Article disponible en ligne à l'adresse :

http://www.cairn.info/revue-etudes-theologiques-et-religieuses-2016-4-page-599.htm

\section{Pour citer cet article :}

Elena Di Pede, «Quelques effets narratifs de la pseudépigraphie jérémienne 》, Études théologiques et religieuses 2016/4 (Tome 91), p. 599-605.

DOI 10.3917/etr.0914.0599

Distribution électronique Cairn.info pour Institut protestant de théologie.

(C) Institut protestant de théologie. Tous droits réservés pour tous pays.

La reproduction ou représentation de cet article, notamment par photocopie, n'est autorisée que dans les limites des conditions générales d'utilisation du site ou, le cas échéant, des conditions générales de la licence souscrite par votre établissement. Toute autre reproduction ou représentation, en tout ou partie, sous quelque forme et de quelque manière que ce soit, est interdite sauf accord préalable et écrit de l'éditeur, en dehors des cas prévus par la législation en vigueur en France. Il est précisé que son stockage dans une base de données est également interdit. 


\section{Quelques effets narratifs de la pseudépigraphie jérémienne}

Dans la version grecque des LXX, la littérature jérémienne ne se limite pas au seul livre qui porte le nom du prophète d'Anatoth. Sous le nom de ce prophète, on trouve également le livre des Lamentations et deux écrits deutérocanoniques, le livre de Baruch et la lettre de Jérémie. Si la question est épineuse du point de vue de la tradition textuelle et de sa transmission, cette trilogie qui suit, et qui tend à compléter JrLXX, introduit à une herméneutique particulière, propre à la tradition grecque. Dans cette brève contribution Elena DI PEDE* explore les effets narratifs de la disposition de la littérature jérémienne dans la tradition de la LXX.

Dans sa version grecque, la littérature jérémienne ne se borne pas au livre du prophète éponyme. Outre ce livre lui-même $\left(\mathrm{JrLXX}^{1}\right)$, on trouvera sous le nom de ce prophète le livre des Lamentations et deux écrits deutérocanoniques, le livre de Baruch et la lettre de Jérémie ${ }^{2}$. La question est ardue du point de vue de la tradition textuelle et de sa transmission, étant donné que Ba et Lm semblent avoir une plus grande affinité narrative avec la version longue de $\mathrm{Jr}^{3}$. Quoi qu'il en soit de cette histoire et de cette affinité, c'est bien dans la LXX que cette tradition est rapportée et c'est donc en son sein qu'il faudra mener l'enquête, car cette « trilogie » qui suit et qui, d'une certaine manière, complète

\footnotetext{
${ }^{*}$ Elena Di PEDE est professeure d'Ancien Testament au département de théologie (CAEPR) à l'université de Lorraine (UFR Sciences Humaines et Sociales - Metz), membre du centre de recherche Écritures (EA 3943).

${ }^{1}$ Sauf mention contraire, dans la suite de cette contribution, les références faites au livre de Jérémie se rapporteront à la $\mathrm{LXX}$.

${ }^{2}$ Dans la suite de cette étude, on utilisera les abréviations pour indiquer le livre, et le nom des personnages en toutes lettres lorsqu'ils sont évoqués en tant qu'acteurs.

${ }^{3}$ Pour reprendre le terme utilisé par Isabelle Assan-Dhôte, Jacqueline MoAtTi-Fine, Baruch, Lamentations, Lettre de Jérémie, Paris, Cerf, coll. « La Bible d'Alexandrie 25.2 », 2008, p. 20.
} 
JrLXX, « ouvre un champ herméneutique propre à cette tradition $»^{4}$. Cette courte étude a pour but d'explorer ce champ d'un point de vue narratif et de suggérer quelques pistes de réflexion concernant les effets de sens d'une telle attribution pseudépigraphe et de la disposition de ces écrits ${ }^{5}$.

\section{UNE DISPOSITION CHRONOLOGIQUE ET GÉOGRAPHIQUE PARTICULIÈRE : DÉCALAGES ET CURIOSITÉS}

En terminant la lecture du livre de Jr, le lecteur de la LXX apprend qu'un horizon de restauration s'ouvre pour le peuple exilé, annonçant ainsi la réalisation des paroles de salut du prophète : le roi en exil, Joaqin (Jékonias), est réhabilité par Ewil-Mérodak (Jr 52,31-34). Cette réhabilitation apparemment sans raison crée la surprise, car elle est plus qu'inattendue après la finale de Jr qui s'achève sur un horizon plutôt bouché. Or, c'est bien sur les possibles raisons de la libération de Joakin que revient le début de Ba, lorsque vingt ans plus tôt, face au roi exilé et au peuple qui l'entoure, Baruch lit un livre qu'il écrit à Babylone et qui n'est autre que celui du prophète dont il a été le fidèle témoin et le secrétaire ${ }^{6}$.

La transition géographique entre la fin de Jr et le début de Ba est lisse : c'est à Babylone que les deux scènes se passent. Mais le début de Ba est clairement un flash-back par rapport à la fin du livre du prophète : environ vingt ans avant la réhabilitation du monarque exilé, Baruch rejoue une scène connue du lecteur : face au peuple et au roi, il lit un livre qui les appelle à la conversion?

${ }^{4}$ Ibid., p. 27-29.

${ }^{5}$ Je ne me pencherai pas ici sur les questions de canon et de transmission textuelle. Pour cela, je renvoie à l'excellente introduction de l'ouvrage précité (ibid., p. 20-41) et aux introductions de chaque écrit individuel (pour Ba : ibid., p. 45-77, pour Lm : ibid., p. 129-189 et pout LtJr : ibid., p. 289-308).

${ }^{6} \mathrm{Je}$ reprends ici à mon compte l'hypothèse avancée par Pierre-Maurice BoGAERT dans plusieurs de ses contributions. Voir en particulier « Le personnage de Baruch et l'histoire du livre de Jérémie. Aux origines du Livre deutérocanonique de Baruch », in Elizabeth A. Livingstone (éd.), Studia Evangelica, VII. Papers presented to the Fifth International Congress on Biblical Studies held at Oxford, 1973, Berlin, Akademie-Verlag, coll. « Texte und Untersuchungen zur Geschichte der Altchristlichen Literatur 126 », 1982, p. 73-81 ; ID., « Les trois formes de Jérémie 52 (TM, LXX et VL) », in Gerard J. Norton, Stephan Pisano (éd.), Tradition of the Text. Studies offered to Dominique Barthélemy in celebration of his 70th birthday, Fribourg-Göttingen, Universitätsverlag-Vandenhoeck \& Ruprecht, coll. « OBO 109 », 1991, p. 1-17. Cette hypothèse est reprise et appuyée narrativement par André WÉNIN, «Y a-t-il un "livre de Baruch" ? À propos du livre récent d'André Kabasele Mukenge », in Jean-Marie Auwers, André Wénin (éd.), Lectures et relectures de la Bible. Festschrift P.-M. Bogaert, Louvain, Peeters, coll. «BETL 144 », 1999, p. 231-243.

\footnotetext{
${ }^{7}$ Sur ces scènes de lecture, voir en particulier André KABASELE MuKEnge, L'unité littéraire du livre de Baruch, Paris, Gabalda, coll. «Études bibliques, Nouvelle Série 38 », 1998, ainsi que son article "Les derniers rois de Juda et la lecture du "Livre" : Josias (2R 22-23), Joiaqim (Jr 36) et Jékonias (Ba 1,1-14) », Revue théologique de Louvain 30 (1999), p. 11-31.
} 
Ce livre, qui contient les paroles de Jérémie, obtient ici un effet inverse à celui qu'il avait suscité lors de sa lecture en Jr 43 : le roi et le peuple se convertissent, expliquant pourquoi Joaqin est réhabilité, signe annonciateur de la fin de l'exil $^{8}$. Le lecteur de Jr ne s'étonnera pas de cette disposition : ce livre est en effet coutumier des flash-back $k^{9}$ qui ont essentiellement pour but d'expliquer les raisons d'une situation particulière. Ce dont le lecteur s'étonnera peut-être davantage c'est de retrouver Baruch à Babylone, alors qu'il l'avait laissé avec le peuple en Égypte (Jr 50,6-7). Mais à bien y réfléchir, sa présence est assez logique. Si la parole prophétique est écrite, il n'est guère besoin du prophète pour qu'elle circule. En revanche, celle de son témoin privilégié est indispensable. En Jr, Baruch est le garant de la réalisation des paroles du prophète ${ }^{10}$, celles qui concernent la restauration (Jr 39,11-15) et celles qui annoncent la destruction (Jr 43). Ces dernières se sont effectivement réalisées : Baruch et les exilés en sont témoins. C'est probablement pour cette raison que la lecture qu'il effectue devant eux, cinq ans après la chute de Jérusalem, provoque les effets de conversion escomptés (Ba 1,5-13). La logique

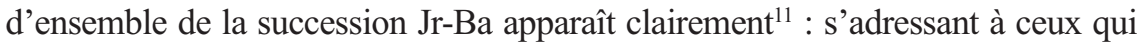
sont en exil et qui, de ce fait, ont d'une certaine manière entendu ou écouté (peutêtre contraints et forcés) la parole de Jérémie, Baruch provoque chez eux une réaction adéquate aux oracles du prophète : la conversion. Celle-ci prend la forme d'une confession collective (1,15-3,8), exprimée dans une liturgie pénitentielle qui ne sera efficace que lorsqu'elle sera réalisée dans la ville sainte ${ }^{12}$. C'est la raison pour laquelle les exilés pénitents envoient à Jérusalem le texte $(1,14$, biblion $)$ de leur confession collective ainsi que l'argent pour couvrir les frais de ce qui est nécessaire au culte (1,6-7), dans l'espoir que par une « confession "vicaire"13 », « les rites célébrés donneront une efficacité à la prière des exilés, dont la pénitence et les supplications en Babylonie n'ont pas suffi à apaiser la colère divine ${ }^{14} »$.

\footnotetext{
${ }^{8}$ En ce sens, voir A. WÉNIN, «Y a-t-il un "livre de Baruch” ?», art. cit., p. 240-241.

${ }^{9}$ Un élément supplémentaire de rapprochement pourrait être le médium littéraire. Comme Jr, en effet, Ba semble être composé en prose (voir les deux premières parties 1,1-14 et 1,15-3,8) et en poésie, ou peut-être en prose rythmée (voir deux parties finales 3,9-4,4 et 4,5-5,9) (I. Assan-DHôTE, J. MoATTIFINE, Baruch, Lamentations, Lettre de Jérémie, op. cit., p. 53-54). Je ne m'attarderai cependant pas sur ce point qui demanderait un travail approfondi dépassant le cadre de cette étude.

${ }^{10}$ Voir Pierre-Maurice BogaERT, «"Vie et paroles de Jérémie selon Baruch". Le texte court de Jérémie (LXX) comme œuvre biographique », in Enzo Bianchi, Vittorio Fusco, Benoît Standaert (éd.), La parola edifica la comunità, Magnano, Edizioni Qiqajon, 1996, p. 15-29.

${ }^{11}$ Et cela, quelles que soient les raisons historiques qui ont mené à l'écriture de Ba et quel que soit son auteur.

${ }^{12} \mathrm{Ba} 1,13 \mathrm{~b}$ semble suggérer que la confession collective à Babylone n'a pas éloigné la colère du Seigneur de « nous ».

${ }^{13}$ A. WÉNIN, «Y a-t-il un "livre de Baruch"? », art. cit., p. 237.

${ }^{14}$ Ibid., p. 236.
} 
Jérusalem, représentée en Ba comme une mère victime des agissements de ses enfants et qui attend le pardon divin afin que ses fils et ses filles puissent revenir à elle (És 60,4), est ainsi au cœur de l'effectivité de la démarche de conversion, car c'est là que tout se joue, pour ses habitants déjà exilés comme pour ceux qui sont encore en son sein. Ba semble ainsi suggérer que le retour à la paix est possible si les deux parties du peuple - les exilés et ceux qui sont encore à Jérusalem collaborent afin d'apaiser le Seigneur. Mais si les exilés, forts de leur expérience, entendent maintenant la parole de Jérémie proclamée par Baruch, les habitants de Jérusalem semblent ne pas l'avoir entendue. Les Lamentations, en effet, reviennent sur la ville désertée par ses habitants (voir le préambule Lm LXX).

Encore une fois, la transition géographique entre la fin de Ba et le début de Lm est lisse, car la version grecque de Lm s'ouvre sur un préambule ${ }^{15}$, absent de l'hébreu, qui situe clairement l'action à Jérusalem, là où le livret envoyé par les exilés avait déjà ramené le lecteur. C'est face à la ville sainte qu'il retrouve maintenant Jérémie, assis en pleurs ${ }^{16}$. Mais si géographiquement la transition est lisse, le lecteur est une fois encore confronté à un flash-back d'au moins cinq ans par rapport aux faits évoqués en Ba, et qui semble situer l'action au moment précis de la prise de la ville et de l'exil de ses habitants (Jr 52). À ce moment-là, constatant la réalisation du malheur qu'il a annoncé pendant bien longtemps sans réussir à ramener le peuple à l'alliance, le prophète n'a plus d'autre ressource que de s'asseoir et de pleurer. Et dans ses larmes, il se lamente sur la ville et ses habitants. De ce point de vue, le contraste avec Ba est saisissant : là c'était les exilés ensemble qui confessaient leur faute en signe de conversion, ici c'est le prophète seul qui constate la débâcle et qui se lamente. Ce contraste est doublé du parallèle qui est construit entre la ville et le prophète : la ville, veuve et déserte $(\mathrm{Lm} \mathrm{1,1)}$ est trahie, bannie par ceux qui étaient ses amis $(\mathrm{Lm} \mathrm{1,2)}$. Elle n'a plus que ses larmes pour pleurer et gémir encore sur son sort et celui de ses enfants. Placé sous le patronage de l'autorité du prophète d'Anathot, Lm met en scène un "serviteur souffrant ", qui porte le deuil et de la ville, et de ses habitants. Cette mise en scène fait de lui comme l'incarnation de ceux- $\mathrm{ci}^{17}$, une personnification qui donne une voix à leurs plaintes et à leurs supplications respectives en « je » ou en «nous».

\footnotetext{
${ }^{15}$ «Et il advint : après qu'Israël eut été emmené en captivité et que Jérusalem fut devenue déserte, Jérémie s'assit en pleurant et proféra cette lamentation sur Jérusalem [...] », trad. I. AsSAN-DHÔTE, J. Moatti-Fine, Baruch, Lamentations, Lettre de Jérémie, op. cit., p. 190.

${ }^{16}$ Ainsi, « Baruch et les Lamentations sont comme deux récits parallèles qui prolongent l'histoire de Jérusalem et la parole de son prophète » (ibid., p. 29).

${ }^{17}$ Ibid., p. 131 ; les auteures parlent à ce propos de mise en abyme. Sur ce point précis et sur la manière dont cette métaphore fonctionne en Jr TM, on verra l'ouvrage de Mario CuCCA, Il Corpo e la Città. Studio del rapporto di significazione paradigmatica tra la vicenda di Geremia e il destino di Gerusalemme, Assise, Cittadella, coll. « Studi e Ricerche, sezione biblica », 2010.
} 
Ce lien étroit entre le prophète et la ville (le sort de l'un étant clairement lié au sort de l'autre) est également présent en Jr, même si dans ce livre leurs sorts apparaissaient plutôt inversés ${ }^{18}$. Mais Lm montre, s'il le fallait encore, d'une part comment le Seigneur veille à la réalisation de ses paroles (voir Jr 1,11-12 et Lm 1,14, avec une correspondance absente en hébreu), et d'autre part que la destruction sur laquelle Jérusalem et Jérémie se lamentent maintenant est le résultat des agissements des contemporains du prophète, une épreuve face à laquelle il faudra réagir ${ }^{19}$. Reste à savoir comment. Car l'exil dont a parlé Ba et face auquel la réaction a été positive, semble n'être que la partie visible de l'iceberg. La destruction et la lamentation qui s'ensuit reprend des thèmes déjà bien présents en Jr : si le peuple est exilé et la ville abandonnée, c'est à cause de leur impiété (Lm 1,14, voir par exemple Jr 5,6;6,7), parce que le peuple s'est tourné vers ses

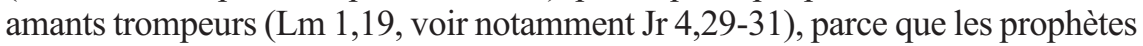
qu'il a écouté n'ont pas « dévoilé [son] injustice » (Lm 2,14, voir entre autres Jr 23,16-22) et qu'il s'est tourné vers les nations en espérant trouver chez elles le salut (Lm 4,18, voir Jr 49-50), autre preuve d'idolâtrie. Ainsi l'exil extérieur n'est que la face visible du véritable exil dans lequel le peuple s'est engouffré, un exil intérieur, duquel le retour au Seigneur est la seule voie de sortie. Mais pour cela, il faut combattre la cause de l'exil, à savoir l'idolâtrie qui persiste dangereusement et sournoisement. Est-ce dès lors étonnant que la plaidoirie qui clôt l'ensemble jérémien revienne sur cette question qui touche tout humain et qui, pour cette raison, n’est jamais réglée définitivement?

\section{LA LETTRE, SATIRE ACERBE CONTRE L'IDOLÂTRIE EN GUISE DE CLÔTURE}

Les jeux sont faits : Jérusalem est tombée et sa population exilée. Pour cela, Israël ne peut s'en prendre qu'à lui-même et porter le deuil, avec le prophète, demandant au Seigneur de le faire revenir, de « renouveler [ses] jours » et de laisser tomber sa colère (Lm 5,21-22) causée par le rejet de l'alliance et le choix de l'idolâtrie assumée par le peuple (voir notamment Jr 10,1-16;38,36 ; 39,19). Il n'est donc pas anodin que, dans un dernier flash-back - le plus long de tous ceux qui ont été disposés jusqu'ici - qui ramène le lecteur auprès de ceux qui s'apprêtent à partir en exil, cette question revienne à l'ordre du jour, comme à suggérer qu'il s'agit de ce qu'il y a de plus fondamental. Car si les destinataires de cette lettre, désormais exilés, se sont convertis (voir Ba), la paix n'est pas revenue pour autant et le danger de l'idolâtrie persiste, tant pour eux que pour le lecteur.

\footnotetext{
${ }^{18}$ La correspondance entre le prophète et la ville est particulièrement bien visible dans la troisième lamentation qui est exprimée presque entièrement à la première personne du singulier.

${ }^{19}$ Le lecteur n'est pas mis face à la lettre elle-même mais à une copie de celle-ci (voir le préambule de la lettre).
} 
Par un double décalage, géographique et temporel, par rapport à ce qui précède, le lecteur est subitement ramené à une autre lettre, celle que Jérémie envoie à ceux qui sont déjà exilés (Jr 36), une lettre antérieure dans la trame jérémienne, mais postérieure pour ce qui est de son envoi - en tout cas dans le monde fictionnel créé par ces écrits. Dans ce monde particulier, Jérémie a donc écrit deux lettres, ce que le lecteur ignorait jusque-là. Après avoir lu la lettre adressée aux exilés, le lecteur prend maintenant connaissance de celle que le prophète a adressée à ceux qui s'apprêtaient à partir ${ }^{20}$. Le Jérémie de la fiction littéraire écrit ainsi contre les faux prophètes et contre l'idolâtrie. D'une certaine manière, ses deux lettres se correspondent ${ }^{21}$ et se complètent. Malgré son style qui est bien loin de celui du livre, en effet, cette deuxième lettre représente une clôture parfaite : elle rappelle à la mémoire du lecteur ce contre quoi Jérémie s'est battu pendant l'ensemble de sa mission - l'idolâtrie -, dans une forme littéraire déjà présente en Jr : une lettre adressée à des concitoyens ${ }^{22}$. Et si celle de Jr 36 invitait les exilés à s'établir tout en les mettant en garde contre les faux prophètes qui les bercent d'illusions, cette fois c'est à la racine du mal que le prophète s'en prend de manière virulente, mais en utilisant le moyen de la satire afin de démontrer le ridicule des faux dieux. Le parallèle est clair entre les deux lettres pour ce qui est de leur contenu : toutes deux mettent en garde contre une illusion mortifère, la parole des faux prophètes d'une part, et les idoles d'autre part. On pourrait même se demander si l'un des critères pour distinguer le vrai prophète du faux n'est pas justement dans le contenu même de son message, le faux prophète étant souvent porteur d'une parole facile à entendre, et par ce fait illusoire. De cette manière c'est cette parole vaine et fausse qui risque de devenir elle-même une idole (voir par exemple la dispute entre Jérémie et Ananias en Jr 35).

Face à l'adresse de cette dernière lettre, le lecteur peut se demander pour quelle raison cette parole n'a pas été directement adressée à ses destinataires, et pourquoi Jérémie leur a écrit plutôt que de leur parler. Le prophète n'était-il pas à Jérusalem à ce moment-là ? Si la question est légitime au regard de la fabula d'ensemble des écrits jérémiens grecs, elle est peut-être superflue à ce stade où tout est joué dans le monde du récit, alors que tout est encore à jouer dans le monde du lecteur. À ce stade, en effet, ce qui compte c'est le contenu de cette lettre qui accompagne les exilés. Il appelle à la louange constante du Seigneur et met perpétuellement en garde contre ce qui éloigne du vrai Dieu, car là est le danger réel en terre d'exil. Le livre de Jr est probablement celui qui illustre le

\footnotetext{
${ }^{20}$ Ce n'est donc pas étonnant, comme le rappellent I. Assan-Dhôte et J. MoatTi-Fine, Baruch, Lamentations, Lettre de Jérémie, op. cit., p. 26 et 290, que l'auteur de la Lettre de Jérémie se glisse dans l'« omission » de Jr 36LXX concernant l'idolâtrie afin d'élaborer son propre texte.

${ }^{21}$ Ibid, p. 292. Les auteures font remarquer que cette lettre ne se conforme pas au modèle de la lettre grecque, mais imite son modèle jérémien.

22 Ibid., p. 303.
} 
mieux cet adage latin : verba volant, scripta manent. Si les mots prononcés par le prophète n'ont pas été entendus, une fois écrits ils ne pourront plus être ignorés aussi facilement. Au-delà des personnages du récit, ils entraînent le lecteur dans un mouvement qui le met face à ses propres responsabilités par rapport à la parole prophétique et à ses appels incessants à retourner à l'alliance et à rejeter les idoles.

La lettre insiste sur l'opposition entre le Seigneur, qu'il faut craindre (Dt 6,13), et les idoles, dont il ne faut pas avoir peur. Cela devient même le refrain de la lettre, à laquelle il imprime un « mouvement d'amplification dans la dénonciation de ce leurre ${ }^{23} \gg$ qu'est l'idolâtrie. En jouant sur les répétitions et les variations subtiles dans l'ordre des mots de ces répétitions, ce refrain martèle les idées qu'il contient et entraîne les destinataires dans son mouvement qui doit aboutir au rejet de ce qui est dénoncé. Et si l'insistance est aussi forte, c'est probablement parce que le lecteur a encore la possibilité de faire changer les choses, contrairement aux destinataires du prophète.

La trilogie de textes qui accompagne le livre de Jérémie dans la LXX réactive par sa disposition même la parole positive de Jérémie, alors que ses oracles de malheurs pour Jérusalem et ses habitants se sont réalisés. Elle pose toutefois question, car elle n'est pas disposée de manière linéaire. Au contraire, elle joue sur des décalages qui peuvent être aussi bien chronologiques que géographiques, dans un balancement qui fait bouger le lecteur dans le temps mais aussi dans l'espace, de Jérusalem à Babylone et de Babylone à Jérusalem. Or, le va-et-vient temporel n'est pas une surprise pour le lecteur de Jr. Déjà au sein même du livre du prophète, la disposition comme en échiquier ${ }^{24}$ des événements entraînait le lecteur dans un approfondissement toujours plus grand des problématiques abordées par le prophète, qui finiront par conduire Israël en exil. La construction de l'ensemble, qui se fait par emboîtements et enchâssements, procède d'un mouvement semblable : reprenant et approfondissant les thématiques abordées dans Jr, les négatives mais surtout les positives, la trilogie insiste sur 1'espoir dans la réalisation des oracles positifs du prophète maintenant que le malheur s'est accompli. Mais le Jérémie de la fiction ne serait pas fidèle à lui-même s'il ne continuait pas à mettre en garde contre les entraves à l'alliance, qui n'auraient d'autre effet que de retarder la réalisation du bonheur annoncé : l'idolâtrie qui, avec le refus d'écouter une parole qui appelle à la vie, est la racine de tout manque d'humanisation.

Elena Di PEDE

\footnotetext{
${ }^{23}$ Joël Rosenberg, « Jérémie et Ézéchiel », in Robert Alter, Frank Kermode (éd.), Encyclopédie littéraire de la Bible, Paris, Bayard, 2003, p. 231-255, voir en particulier p. 242.
} 